\title{
Novedades florísticas en la vertiente norte de Sierra Nevada (Granada, España)
}

\author{
Manuel Pavón Núñez, Noelia Hidalgo Triana \& Andrés Vicente Pérez Latorre \\ Departamento de Botánica y Fisiología Vegetal. Facultad de Ciencias. Universidad de Málaga. Málaga. España.
}

\section{Correspondencia}

M. Pavón Núñez

e-mail: manuelp1764@gmail.com

Recibido: 5 noviembre 2019

Aceptado:2 marzo 2020

Publicado on-line: 1 junio 2020

Editado por: Antonio Galán de Mera

\begin{abstract}
Resumen
Se presenta un listado de 14 taxones que son novedosos o de interés para Andalucía y que han sido recolectados en la cara norte de Sierra Nevada (Granada). Se incluyen datos de la biología, corología y ecología de todos ellos, además de algunas fotos de especies significativas.
\end{abstract}

Palabras clave: Flora vascular, corología, Sierra Nevada, Granada, Andalucía oriental.

\begin{abstract}
New records on the flora of the northern face of Sierra Nevada (Granada, Spain)

In this paper, 14 new or interesting taxa are listed for Andalusia and have been collected on the north face of Sierra Nevada (Granada). Biology, chorology and ecology data of all of them are included, as well as some photographs of significant species.
\end{abstract}

Key words: Vascular flora, chorology, Sierra Nevada, Granada, Eastern Andalusia
Con este trabajo, se pretende dar a conocer las citas que resultan novedades corológicas, confirmación de presencias y/o nueva información sobre taxones de interés a partir del catálogo resultante de las prospecciones botánicas que se están realizando, como parte de una tesis doctoral, en la cuenca alta del río Alhama de Lugros (Dehesa del Camarate) (Pavón et al., 2019). La zona en estudio se encuentra dentro del Parque Nacional de Sierra Nevada, (Granada, España) (Fig. 1) y adscrita biogeográficamente al sector Nevadense, distrito Nevadense de la provincia Bética en la región Mediterránea (Marfil et al., 2016). Las herborizaciones se vienen realizando desde agosto de 2018, quedando depositado el material recolectado en el Herbario MGC de la Universidad de Málaga. Para su identificación y nomenclatura, se han consultado las obras Flora iberica (Castroviejo et al., 1986-2019) y Flora de Andalucía Oriental (Blanca et al., 2011). También se ha tenido en cuenta la lista actualizada de flora vascular de Sierra Nevada (Lorite, 2016). Para las comprobaciones de las novedades corológicas se ha utilizado la base de datos ANTHOS (2019) y para las verificaciones fitocenológicas se ha seguido a Rivas-Martínez (2011) y la base de datos SIVIM (2019). Las novedades en lo referente a pliegos de herbario se han confirmado mediante búsquedas en bases de datos de biodiversidad vegetal, fundamentalmente GBIF (2019).

Los taxones que se han seleccionado en este artículo son novedades para un subsector/distrito biogeográfico o para las comarcas de Andalucía Oriental, o bien representan una nueva población de especies protegidas (Decreto 23/2012, 14 febrero, Junta de Andalucía) o amenazadas (Cabezudo et al., 2005a).

Como resultado, se aporta un listado de 14 taxones, ordenados alfabéticamente. De cada taxón se describen los datos correspondientes al biotipo, corología general, ecología general y local (Castroviejo et al., 1986-2019 y Blanca et al., 2011). En cuanto a novedades como citas con pliego de herbario (GBIF), se dan a conocer: un taxón para la mitad sur de España (Epilobium montanum L.), dos para la provincia de Granada (Moehringia trinervia (L.) Clairv. y Geranium sylvaticum L.), seis para el Parque Nacional de Sierra Nevada (Cota triumfettii (L.) J. Gay, Epilobium lanceolatum Sebast. \& Mauri, Lepidium villarsii Gren. \& Godr. subsp. villarsii, Gagea lacaitae A. Terrac., Sedum forsterianum Sm. y Sorbus aucuparia L.), y una novedad para el distrito Nevadense (Elymus hispanicus (Boiss.) Talavera). Como nuevas poblaciones de especies protegidas/amenazadas, se citan tres taxones (Ribes uva-crispa L. subsp. austro-europaeum 


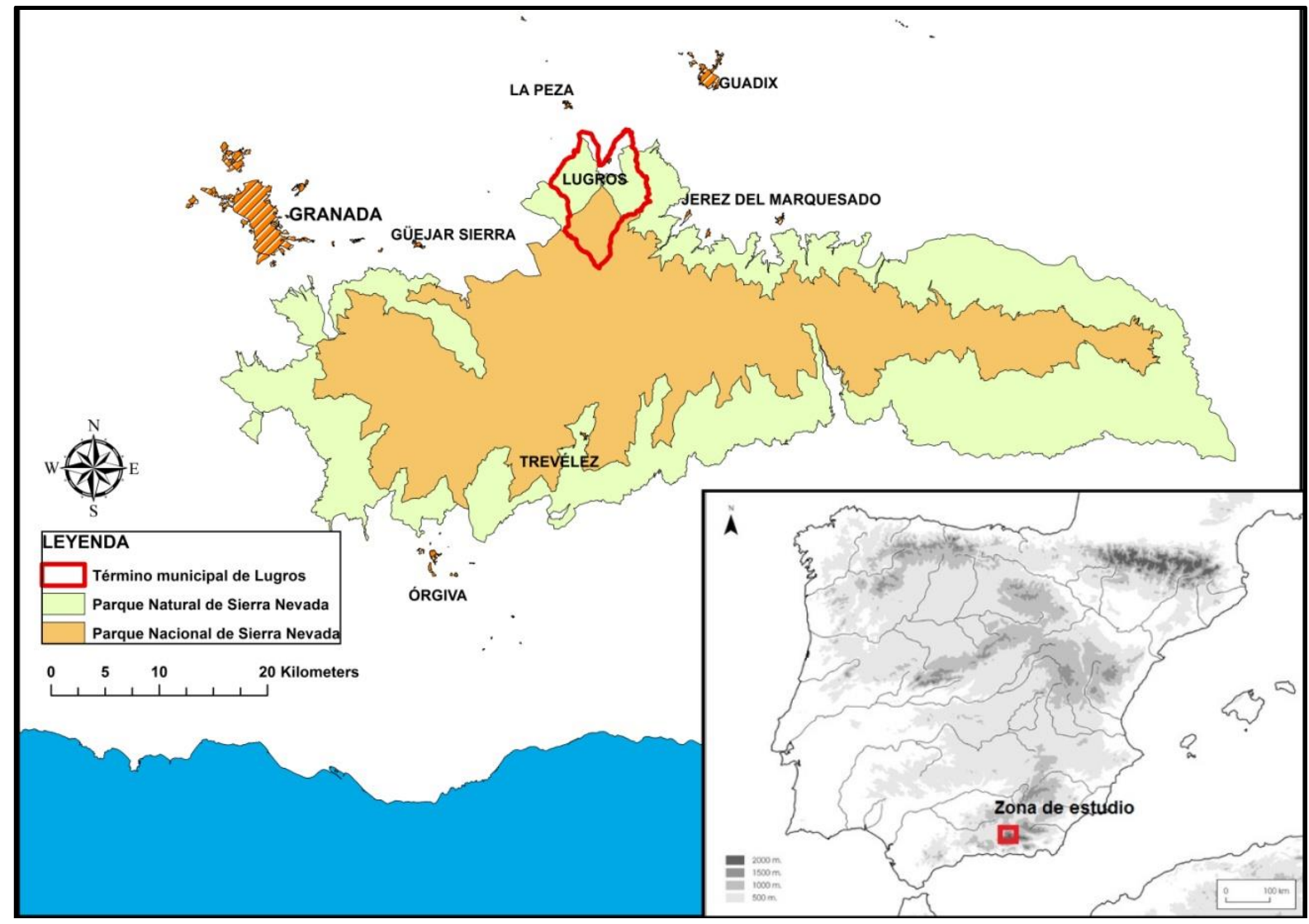

Figura1. Localización geográfica de la zona de estudio. Fuente: elaboración propia a partir de coberturas disponibles en DERA (2019).

Figure1. Geographical location of the study area. Source: Own elaboration using the coverage available in DERA (2019).

(Bornm.) Bech., Sarcocapnos crassifolia (Desf.) DC subsp. speciosa Boiss. y Ophioglossum vulgatum L.)

Cota triumfettii (L.) J. Gay, FI. Sicul. Syn. 2: 867 (1855)

[= Anthemis triumfettii All., Fl. Pedem. : 187 (1785)]

ESPAÑA. Granada: Lugros, Dehesa del Camarate, 30S476992/4115745, 1400-1457 m., $15 / 06 / 2019$, Pastizal de lindero de melojar mixto nevadense, cambisol, micaesquistos y cuarcitas, $M$. Pavón Núñez, A.V. Pérez Latorre \& N. Hidalgo (MGC 91692).

Hemicriptófito erecto. Submediterránea. Europa y Asia Menor. En Andalucía, se encuentra en las provincias de Jaén y Granada, fundamentalmente en las Sierras de Cazorla y Segura y Sierra Nevada (Blanca, 2011a). Los individuos localizados en la Dehesa del Camarate, representan la primera cita con pliego de herbario (GBIF) para el Parque Nacional de Sierra Nevada, de donde se conocían algunas referencias bibliográficas (Molero Mesa \& Martínez Parras, 1983; Molero Mesa \& Pérez Raya, 1987; Lorite, 2016). La especie recolectada forma parte de pastizales esciófilos vivaces de lindero de bosques caducifolio-marcescentes (TrifolioGeranietea Müller 1962) en el dominio del melojar mixto (Adenocarpo-Quercetum pyrenaicae aceretosum granatensis Martínez Parras \& Molero Mesa 1982), en el termotipo supramediterráneo y sobre suelos profundos, ricos en materia orgánica, derivados de micaesquistos y cuarcitas, tipo cambisol eútrico.

Elymus hispanicus (Boiss.) Talavera in Lagascalia 14(1): 170 (1986)

ESPAÑA. Granada: Lugros, Dehesa del Camarate, 30S476992/4115745, 1400-1457 m., 15/06/2019, Pastizal de lindero de melojar mixto nevadense, cambisol, micaesquistos y cuarcitas, $M$. Pavón Núñez, A.V. Pérez Latorre \& N. Hidalgo (MGC 91668).

Hemicriptófito cespitoso. Íbero-Magrebí. Pastizales esciófilos bajo bosquetes caducifolios y marcescentes. En Andalucía está presente a lo largo de las Sierras Béticas (Negrillo, 2011), donde está ampliamente citada; las poblaciones más próximas son las de las sierras de Tejeda y Almijara (Cabezudo et al., 2005b; Pavón Núñez et al., 2015). De Sierra Nevada, existen dos citas antiguas de herbario (BC 815316, BC 815317) y algunas citas bibliográficas (Boissier, 1839; Lorite, 2016), en la zona de Monachil y Trevenque (proximidades al Cortijo de Cortichuela), sector Almijaro-Granatense (Pérez Latorre et al., 2004). En la Dehesa del Camarate, y por tanto en el distrito Nevadense, es primera cita. Ha sido recolectada en pastizales vivaces de lindero de bosque (Origanetalia vulgaris Müller 1962), en el dominio del melojar mixto 
nevadense (Adenocarpo-Quercetum pyrenaicae aceretosum granatensis), en el termotipo supramediterráneo y en suelos profundos y frescos derivados de micaesquistos y cuarcitas, tipo cambisol eútrico.

Epilobium lanceolatum Sebast. \& Mauri, FI. Rom. Prod.: 138, tab. 1 (1818)

(Fig. 2D)

ESPAÑA. Granada: Lugros, Dehesa del Camarate, 30S476992/4115745, 1400-1460 m., 15/06/2019, Pastizal de lindero de melojar mixto nevadense, cambisol, micaesquistos y cuarcitas, $M$. Pavón Núñez, A.V. Pérez Latorre \& N. Hidalgo (MGC 91717).

Hemicriptófito erecto. Circunmediterránea y atlántica. En bosques, herbazales y roquedos húmedos, preferentemente silíceos. Europa occidental y meridional, Asia y Norte de África. Mitad norte y oeste de la Península y Sierra Nevada (Nieto Feliner, 1997). Existen citas y referencias bibliográficas en las provincias de Cádiz, Granada y Málaga (Rigueiro Rodríguez, 1978; Lorite, 2016; Casimiro-Soriguer et al., 2019). Hasta ahora, existía una cita de herbario (GBIF) en la provincia de Málaga (MGC 52287-1) y otra en la provincia de Cádiz (MA 774153-1), a las que hay que añadir tres citas nuevas con pliego de herbario (GBIF) en la provincia de Málaga, Sierra de las Nieves (MGC 89598, MGC 90145, MGC 90146). Es la primera cita con pliego de herbario (GBIF) para el Parque Nacional de Sierra Nevada. Ha sido localizada en un pastizal húmedo de fondo de barranco umbroso, en el lindero de melojar mixto (Adenocarpo-Quercetum pyrenaicae aceretosum granatensis), en el termotipo supramediterráneo y en suelos profundos y frescos derivados de micaesquistos y cuarcitas, tipo cambisol eútrico.

\section{Epilobium montanum L., Sp. PI.: 348 (1753)}

ESPAÑA. Granada: Lugros, Dehesa del Camarate, 30S476992/4115745, 1690-1720 m., 17/07/2019, Pastizal edafohigrófilo de bosque mixto caducifolio, fluvisol, micaesquistos y cuarcitas, $M$. Pavón Núñez \& A.V. Pérez Latorre (MGC 92326).

Hemicriptófito erecto. Paleotemplada. Pastizales higrófilos de montaña ricos en materia orgánica. Regiones templadas de Europa y Asia. Mitad norte de España y Sierra Nevada (Nieto Feliner, 1997). Aunque existían dos referencias bibliográficas en Sierra Nevada (Molero Mesa \& Pérez Raya, 1987; Lorite, 2016), es la primera cita con pliego de herbario (GBIF) para la mitad sur de la Península Ibérica. En la Dehesa del Camarate, esta especie ha sido recolectada en un pastizal edafohigrófilo de borde de arroyo de aguas permanentes (MolinioArrhenatheretea Tüxen 1937) que transcurre bajo el dosel de un bosque mixto caducifolio (Fagetalia sylvaticae Pawłowski in Pawłowski, Sokołowski \& Wallisch 1928), en el termotipo supramediterráneo y sobre suelos profundos derivados de micaesquistos y cuarcitas, tipo fluvisol.

Fraxinus excelsior L., Sp. Pl.: 1057 (1753)
ESPAÑA. Granada: Lugros, Dehesa del Camarate, 30S476992/4115745, 1400-1430 m., $18 / 05 / 2019$, Bosque mixto caducifolio de ribera, fluvisol, micaesquistos y cuarcitas, M. Pavón Núñez, A.V. Pérez Latorre \& N. Hidalgo (MGC 90704).

Mesofanerófito caducifolio. Eurosiberiana. Bosques caducifolios climatófilos y edafohigrófilos. Europa y Oeste de Asia. Norte, Centro y Este de la Península Ibérica (Andrés, 2012). Existen diversas citas y referencias bibliográficas a lo largo de las Cordilleras Béticas (Sánchez Gómez et al., 2005; Cabezudo et al., 2011). En la Dehesa del Camarate, existía una única cita de herbario (GBIF) antigua (MA 751674, MA 751675) y también una cita bibliográfica (Prieto, 1975). Representa la segunda cita con pliego de herbario (GBIF) para el Parque Nacional de Sierra Nevada y también para la zona de estudio. Ha sido recolectado en un bosque caducifolio mixto de ribera (Populetalia albae $\mathrm{Br}$.-BI. ex Tchou 1948), en el termotipo supramediterráneo y en suelos profundos derivados de micaesquistos $y$ cuarcitas, tipo fluvisol.

Gagea lacaitae A. Terracc. in Boll. Soc. Ortic. Palermo 2(4): 60 (1904)

ESPAÑA. Granada: Lugros, Dehesa del Camarate, 30S476992/4115745, 1850-1905 m., 15/04/2019, Pastizal orófilo, regosol, micaesquistos y cuarcitas, M. Pavón Núñez \& A.V. Pérez Latorre (MGC 90326).

Geófito bulboso. Mediterránea occidental. Vegetación pratense orófila. En Andalucía se localiza en las provincias de Almería, Granada, Jaén y Málaga (López González, 2013). En Sierra Nevada existe una cita bibliográfica (Lorite, 2016) y otras citas próximas se encuentran en Sierra de Baza (Ferrer Gallego \& Guara Requena, 2007) y en Sierra de Gádor (Blanca \& Morales, 1991) en matorrales, pastizales y sabinares de alta montaña. La cita de herbario (GBIF) más próxima se encuentra en Játar, Sierra de Almijara (MGC 66673). Se trata de la primera cita de herbario (GBIF) para Sierra Nevada. El biótopo donde ha sido recolectada esta especie, similar a los mencionados, se corresponde con pastizales del piso oromediterráneo inferior, pastoreados y de suelos profundos crioturbados (Sideritido fontquerianae-Arenarion microphyllae Rivas Goday \& Borja 1961) de zonas abiertas del piornal nevadense (Genisto versicoloris-Cytisetum nevadensis Rivas-Martínez \& Molero in RivasMartínez et al. 2011), sobre suelos de tipo regosol dístrico derivados de rocas silíceas como micaesquistos y cuarcitas.

Geranium sylvaticum L., Sp. Pl.: 681 (1753) (Fig. 2B)

ESPAÑA. Granada: Lugros, Dehesa del Camarate, 30S476992/4115745, 1690-1717 m., 18/05/2019, Pastizal escionitrófilo de bosque caducifolio, cambisol, micaesquistos y cuarcitas, $M$. Pavón Núñez, A.V. Pérez Latorre \& N. Hidalgo (MGC 90723).

Hemicriptófito erecto. Eurosiberiana. Pastizales escionitrófilos de bosques caducifolios. Europea y 
asiática. Se encuentra en la mayoría de las montañas de la mitad norte peninsular y en las sierras de Alcaraz, Segura y Sierra Nevada (Aedo, 2015). En Andalucía, eran conocidas las citas de herbario (GBIF) y referencias bibliográficas pertenecientes a la Sierra de Segura (Soriano, 1983; Valle et al., 1988; Ríos Ruiz et al., 2003; Pavón Núñez \& Pérez Latorre, 2010). En el Parque Nacional de Sierra Nevada existen varias citas bibliográficas sin pliego de herbario (GBIF) (Socorro, 1982; Molero Mesa \& Pérez Raya, 1987; Lorite, 2016), por lo que es novedad para Sierra Nevada, para la provincia de Granada y para el sector Nevadense. En la zona de estudio, ha sido recolectado en un pastizal escionitrófilo nemoral (Galio-Alliarion petiolatae Oberdorfer \& Lohmeyer in Oberdorfer et al., 1967), en el dominio del melojar mixto (Adenocarpo-Quercetum pyrenaicae aceretosum granatensis), en el termotipo supramediterráneo y sobre suelos profundos, ricos en materia orgánica, derivados de micaesquistos y cuarcitas, de tipo cambisol.

Lepidium villarsii Gren. \& Godr., Fl.France 1: 150 (1847) subsp. villarsii

[= L. villarsii subsp. reverchonii (Debeaux) Breistr. in Bull. Soc. Iseré 61: 640 (1947)]

ESPAÑA. Granada: Lugros, Dehesa del Camarate, 30S476992/4115745, 1700-1776 m., 18/05/2019, Pastizal edafohigrófilo de bosque mixto caducifolio, fluvisol, micaesquistos y cuarcitas, $M$. Pavón Núñez \& A.V. Pérez Latorre (MGC 90754).

Hemicriptófito cespitoso. Alpina. Pastizales higrófilos de montaña. Alpes, Pirineos y montañas del norte, este y sureste peninsular (Hernández Bermejo \& Clemente, 1993). En Andalucía, las citas y referencias bibliográficas más cercanas pertenecen a las Sierras de Filabres y Baza. En el primer caso se trata de una cita con pliego de herbario (GBIF, HUAL 595-1); en el segundo, es una cita bibliográfica sin pliego de herbario (Navarro et al., 2007). Representa la segunda cita con pliego de herbario (GBIF) para Andalucía y primera para el Parque Nacional de Sierra Nevada (y el distrito Nevadense). Ha sido localizada en un pastizal edafohigrófilo de arroyo permanente de montaña (Molinio-Arrhenatheretea), bajo bosquetes mixtos caducifolios (Fagetalia sylvaticae), en el termotipo supramediterráneo superior y sobre suelos profundos derivados de micaesquistos y cuarcitas, tipo fluvisol.

Moehringia trinervia (L.) Clairv., Man. Herbor. Suisse: 150 (1811) (Fig. 2C)

ESPAÑA. Granada: Lugros, Dehesa del Camarate, 30S476992/4115745, 1400-1457 m., 15/06/2019, Pastizal nemoral de melojar mixto nevadense, cambisol, micaesquistos y cuarcitas, $M$. Pavón Núñez, A.V. Pérez Latorre \& N. Hidalgo (MGC 91702).

Terófito/hemicriptófito reptante. Eurosiberiana. Pastizal nemoral de bosques húmedos caducifolios. Europa, Asia y Norte de África. Mitad norte de la
Península lbérica, rara en las montañas de la mitad sur (Monserrat Martí, 1990). Hasta ahora, existía una única cita de herbario (GBIF) en la mitad sur de España (MGC 72188-1) y algunas referencias bibliográficas que corresponden a la Sierra de Segura, en la provincia de Jaén (Pavón \& Pérez Latorre, 2010; Cabezudo et al., 2010). En Sierra Nevada, se conocían algunas citas bibliográficas (Molero Mesa \& Pérez Raya, 1987). Es novedad para la provincia de Granada y segunda cita con pliego de herbario para la mitad sur de España. Ha sido recolectada en un pastizal escionitrófilo (GalioAlliarion petiolatae), en el dominio del melojar mixto (Adenocarpo-Quercetum pyrenaicae aceretosum granatensis), en el termotipo supramediterráneo y sobre suelos profundos, húmedos, ricos en materia orgánica, derivados de micaesquistos y cuarcitas, tipo cambisol eútrico.

Ophioglossum vulgatum L., Sp. Pl.: 1062 (1753) (Fig. 2A)

ESPAÑA. Granada: Lugros, Dehesa del Camarate, 30S476992/4115745, 1700-1776 m., 25/07/2018, Pastizal edafohigrófilo de bosque mixto caducifolio, fluvisol, micaesquistos y cuarcitas, $M$. Pavón Núñez, A.V. Pérez Latorre \& N. Hidalgo (MGC 89223).

Geófito rizomatoso. Holártica. Pastizales edafohigrófilos. Europa, Norte de Asia y Norte de América, alcanzando también la parte septentrional de África. Mitad norte y oeste de la Península y Sierra Nevada (López González, 1986). Taxón catalogado en la Lista Roja de la Flora Vascular de Andalucía como en peligro crítico CR (Cabezudo et al., 2005a). En Sierra Nevada, existen referencias bibliográficas de la especie (Varo \& Salvo, 1982; Molero Mesa \& Pérez Raya, 1987; Morales \& Fernández Casas, 1989; Lorite 2016). En Andalucía, existen dos citas de herbario (GBIF): una en Sierra Nevada, en Haza del Cerezo (Capileira, vertiente sur de Sierra Nevada, ABH 13660) y otra en Sierra de Segura, en Arroyo de la Balasna (Siles, MUB 100182). Por tanto, se trata de la tercera cita de herbario para Andalucía, la segunda para el Parque Nacional de Sierra Nevada y primera para la zona de estudio. En la Dehesa del Camarate, ha sido localizada en un pastizal edafohigrófilo (MolinioArrhenatheretea) de arroyo de montaña de aguas permanentes, bajo bosquetes mixtos caducifolios (Fagetalia sylvaticae), en el termotipo supramediterráneo superior y sobre suelos profundos derivados de micaesquistos y cuarcitas, tipo fluvisol.

Ribes uva-crispa L. subsp. austro-europaeum

(Bornm.) Bech. in Repert. Spec. Nov. Regni Veg. 25: 228 (1929)

ESPAÑA. Granada: Lugros, Dehesa del Camarate, 30S476992/4115745, 1850-1905 m., 15/04/2019, Espinar caducifolio, cambisol, micaesquistos y cuarcitas, M. Pavón Núñez \& A.V. Pérez Latorre (MGC 90327). 
Nanofanerófito caducifolio. Euroasiática. Europa, desde la Península Ibérica hasta Asia central y China, alcanzando el norte de África. Espinares de orla de bosques caducifolios climatófilos. En Andalucía, se encuentra en las provincias de Almería, Granada, Jaén (Blanca, 1997; Blanca, 2011b) y Málaga (Casimiro-Soriguer Solanas et al., 2019). En Sierra Nevada, se trata de una especie con dos núcleos poblacionales principales, el más numeroso, en el entorno del río Vadillo, con unos 500 ejemplares y otro núcleo menor en la Dehesa del Camarate, con cinco individuos dispersos (Lorite et al., 2005). Taxón catalogado a nivel autonómico en la Lista Roja de la flora vascular como vulnerable VU (Cabezudo et al., 2005). En la zona estudiada, se han contabilizado para este trabajo el número total de individuos, que alcanza los 58 ejemplares. Forman parte del espinar caducifolio de orla (Lonicero arboreae-Berberidion hispanicae O. Bolòs 1954), bajo el dominio de bosques planifolios mixtos de ladera y barrancos, (Fagetalia sylvaticae), en el termotipo oromediterráneo inferior, sobre suelos profundos y frescos, tipo cambisol eútrico, derivados de rocas silíceas, micaesquistos y cuarcitas.

Sarcocapnos crassifolia (Desf.) DC. subsp. speciosa (Boiss.) Rouy in Bull. Soc. Bot. France 31: 53 (1884) (Fig. 2E)

ESPAÑA. Granada: Lugros, Dehesa del Camarate, 30S476992/4115745, 1680-1717 m., 18/05/2019, Grietas de farallón silíceo, leptosol, micaesquistos y cuarcitas, M. Pavón Núñez, A.V. Pérez Latorre \& N. Hidalgo (MGC 90730).

Caméfito sufruticoso. Vegetación casmofítica de paredones verticales y extraplomados. Sierras del sureste peninsular. Se encuentra en las provincias de Almería, Granada y Jaén (Lidén, 1986). Taxón protegido por la legislación autonómica (Decreto 23/2012, 14 febrero, Junta de Andalucía) con la categoría de EN y por la legislación estatal con la misma categoría (Real Decreto 139/2011). También catalogado en la Lista Roja de la Flora Vascular de Andalucía como vulnerable VU (Cabezudo et al., 2005a). Ampliamente citada en la provincia de Granada y en Sierra Nevada, existen citas y referencias bibliográficas (Morales Abad, 1990; Morales \& Romero-García, 1991; Peñas et al., 2005; Pérez-García et al., 2007; Lorite, 2016). Se trata de una población nueva de una especie protegida y novedad para la zona de estudio, donde representa la primera cita con pliego de herbario (GBIF). Está localizada en fisuras terrosas de paredones rocosos orientados al norte (Sarcocapnion pulcherrimae F. Casas 1972 corr. Rivas-Martínez et al., 2002), en el termotipo supramediterráneo y sobre micaesquistos y cuarcitas.
Sedum forsterianum Sm., Engl. Bot. 26: tab. $1802(1807)$

ESPAÑA. Granada: Lugros, Dehesa del Camarate, 30S476992/4115745, 1400-1465 m., $15 / 06 / 2019$, Taludes pedregosos de lindero del melojar mixto, regosol, micaesquistos y cuarcitas, $M$. Pavón Núñez, A.V. Pérez Latorre \& N. Hidalgo (MGC 91686).

Caméfito suculento. Taludes pedregosos y arenosos en suelos silíceos, principalmente en robledales de Quercus pyrenaica. Europa occidental. Dispersa en la Península lbérica, rara en el NE (Castroviejo \& Velayos, 1997). Presente en casi todas las provincias andaluzas, es más frecuente en la zona oriental. En Sierra Nevada, existen citas y referencias bibliográficas (Martínez Parras et al., 1985; Molero Mesa \& Pérez Raya, 1987; Lorite, 2016). Es novedad como cita con pliego de herbario (GBIF) para el Parque Nacional de Sierra Nevada. Se ha recolectado en los taludes umbrosos de lindero de bosque mixto caducifolio (AdenocarpoQuercetum pyrenaicae aceretosum granatensis), en el termotipo supramediterráneo, en suelos silíceos derivados de micaesquistos y cuarcitas, tipo regosol.

Sorbus aucuparia L., Sp. Pl.: 477 (1753)

ESPAÑA. Granada: Lugros, Dehesa del Camarate, 30S476992/4115745, 1400-1450 m., 17/07/2019, Lindero del melojar mixto, cambisol, micaesquistos y cuarcitas, M. Pavón Núñez \& A.V. Pérez Latorre (MGC 92305).

Mesofanerófito caducifolio. Eurosiberiana. Márgenes y claros de bosques caducifolios, abetales y pinares de montaña. Europa y Asia. Montañas de la mitad norte de la Península Ibérica, rara en Sierra Nevada y La Sagra (Aedo \& Aldasoro, 1998). Taxón catalogado en la Lista Roja de la Flora Vascular de Andalucía como EX (Cabezudo et al., 2005a). Se encuentra incluido en el listado andaluz de especies silvestres en régimen de protección especial (Decreto 23/2012). En la mitad sur de la Península se conoce una única cita de herbario (GBIF) en la Sierra de Cazorla (COA 19182-1). En Sierra Nevada, existen diversas referencias bibliográficas (Rivas-Martínez, 1961; Prieto, 1975; Molero Mesa \& Pérez Raya, 1987; Salazar, 1996; Pérez Latorre et al., 2011). Se trata de la segunda cita con pliego de herbario (GBIF) para la mitad sur peninsular y primera para el Parque Nacional de Sierra Nevada. Se han localizado dos individuos juveniles, únicamente con caracteres vegetativos (se han utilizado yemas y brotes para su identificación). Habitan en una zona abierta del bosque mixto caducifolio (Fagetalia sylvaticae), en el termotipo supramediterráneo, en suelos profundos y frescos derivados de micaesquistos y cuarcitas, tipo cambisol eútrico. 


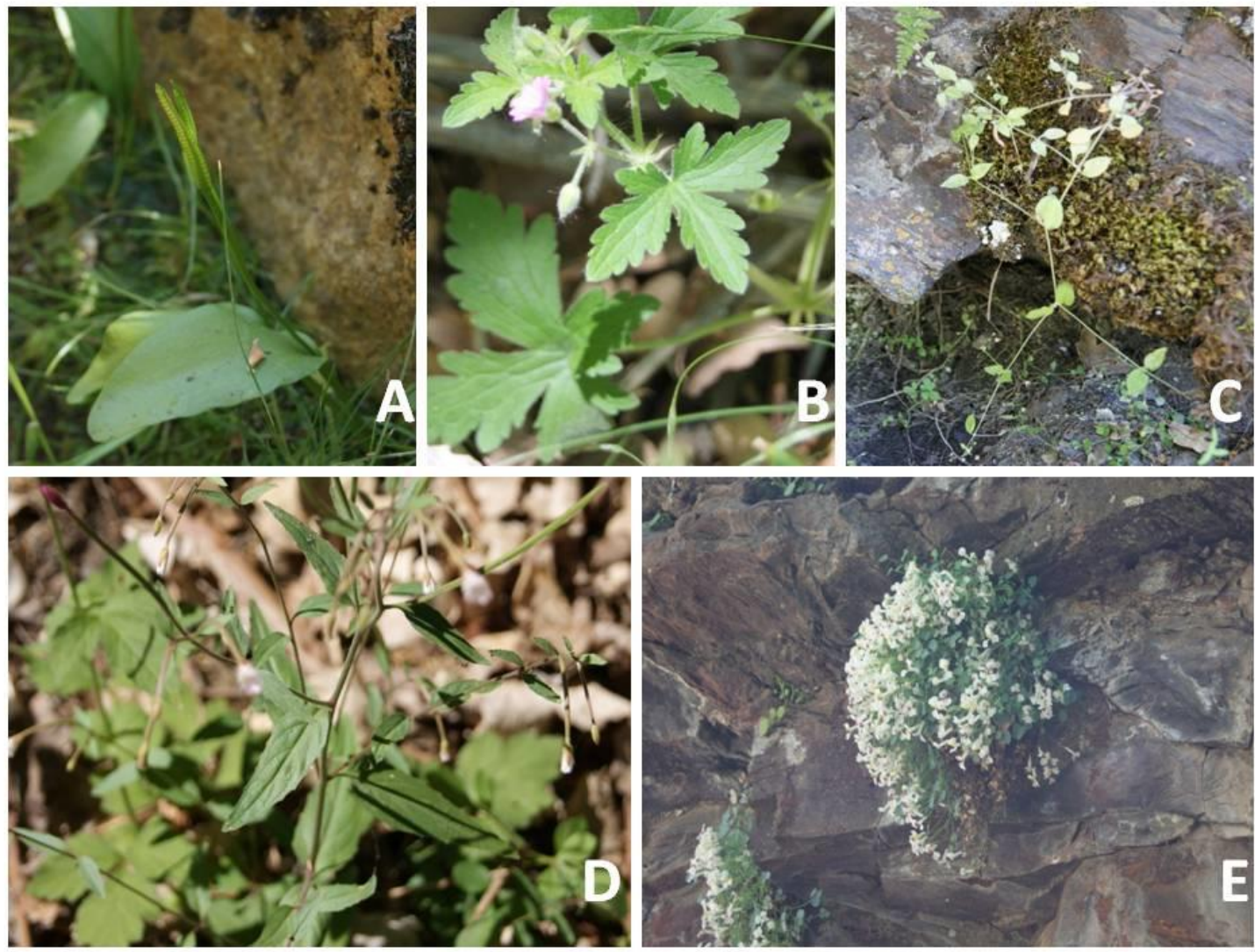

Figura 2. Fotografías de algunas plantas significativas de la vertiente norte de Sierra Nevada. A: Ophioglossum vulgatum L. (Barranco de las Rozas), B: Geranium sylvaticum L. (Barranco de las Rozas), C: Moehringia trinervia (L.) Clairv. (Dehesa del Camarate), D: Epilobium lanceolatum Sebast. \& Mauri (Dehesa del Camarate), E: Sarcocapnos crassifolia (Desf.) DC subsp. speciosa Boiss. (Dehesa del Camarate).

Figure 2. Some photographs of significant plants from the northern face of Sierra Nevada. A: Ophioglossum vulgatum $L$. (Barranco de las Rozas), B: Geranium sylvaticum L. (Barranco de las Rozas), C: Moehringia trinervia (L.) Clairv. (Dehesa del Camarate), D: Epilobium lanceolatum Sebast. \& Mauri (Dehesa del Camarate), E: Sarcocapnos crassifolia (Desf.) DC subsp. speciosa Boiss. (Dehesa del Camarate).

\section{Agradecimientos}

A la dirección y personal del Parque Nacional de Sierra Nevada por los permisos necesarios para la investigación y por la colaboración de los agentes de medio ambiente. Este trabajo se enmarca en una tesis doctoral bajo la cobertura del Grupo de Investigación RNM-115 de la Universidad de Málaga para el estudio funcional y geobotánico de los bosques mixto-caducifolios relictos en Sierra Nevada.

\section{Bibliografía}

Aedo, C. (2015). Geranium L. In F. Muñoz Garmendia, C. Navarro, A. Quintanar \& A. Buira (Eds.), Flora iberica 9 (pp. 271-315). Madrid, España: CSIC.

Aedo, C. \& Aldasoro, J. J. (1998). Sorbus L. In F. Muñoz Garmendia \& C. Navarro (Eds.), Flora iberica 6 (pp. 414-429). Madrid, España: CSIC.

Andrés, C. (2012). Fraxinus L. In S. Talavera, C. Andrés, M. Arista, M. P. Fernández Piedra, M. J.
Gallego, P. L. Ortiz, C. Romero Zarco, F. J. Salgueiro, S. Silvestre \& A. Quintanar (Eds.), Flora iberica 11 (pp. 144-151). Madrid, España: CSIC.

Anthos. [2019]. Sistema de información de plantas de España. Real Jardín Botánico, CSICFundación Biodiversidad. Recurso electrónico en www.anthos.es. Consulta realizada en agosto de 2019.

Blanca, G. (1997). Ribes L. In S. Castroviejo, C. Aedo, M. Laínz, R. Morales, F. Muñoz Garmendia, G. Nieto Feliner \& J. Paiva (Eds.), Flora iberica 5 (pp. 87-94). Madrid, España: CSIC.

Blanca, G. (2011a). Cota (L.) J. Gay. In G. Blanca, B. Cabezudo, M. Cueto, C. Morales Torres \& C. Salazar (Eds.), Claves de la Flora Vascular de Andalucía Oriental (pp. 609). Granada, España: Universidades de Almería, Granada, Jaén y Málaga.

Blanca, G. (2011b). Ribes L. In G. Blanca, B. Cabezudo, M. Cueto, C. Morales Torres \& C. Salazar (Eds.), Claves de la Flora Vascular de Andalucía Oriental, (pp. 250-251). Granada, 
España: Universidades de Almería, Granada, Jaén y Málaga.

Blanca, G., Cabezudo, B., Cueto, M., Salazar, C. \& Morales Torres, C. (Eds.). (2011). Flora Vascular de Andalucía Oriental, $2^{\mathrm{a}}$ edición. Granada, España: Universidades de Almería, Granada, Jaén y Málaga.

Blanca, G. \& Morales, C. (1991). Flora del Parque Natural de la Sierra de Baza. Granada, España: Universidad de Granada.

Boissier, E. (1839). Voyage botanique dans le midi de L'Espagne pendant l'anne 1837. Paris, Francia: Gide et Cie.

Cabezudo, B., S. Talavera, G. Blanca López, C. Salazar, M. Cueto, B. Valdés, J.E. Hernández Bermejo, C. M. Herrera, M. C. Rodríguez Hiraldo \& D. Navas (2005a). Lista roja de la flora vascular de Andalucía. Sevilla, España: Consejería de Medio Ambiente. Junta de Andalucía.

Cabezudo, B., Pérez Latorre, A.V., Navas Fernández, D., Gavira, O. \& Caballero, G. (2005b). Contribución al conocimiento de la flora del Parque Natural de las Sierras Tejeda, Almijara y Alhama (Málaga-Granada, España). Acta Botanica Malacitana, 30, 55-110.

Cabezudo, B., Pérez Latorre, A.V., CasimiroSoriguer Solanas, F., Gavira, O., García Rojas, J.A. \& Pavón, M. (2010). Nota sobre Abies pinsapo Clemente ex Boiss. y otras plantas andaluzas. Acta Botanica Malacitana, 35, 195204.

Cabezudo, B., Pérez Latorre, A.V., CasimiroSoriguer Solanas, F., Pavón, M. \& Hidalgo Triana, N. (2011). Algunas plantas interesantes de Andalucía Oriental (Málaga-Granada). Acta Botanica Malacitana, 36, 213-217.

Casimiro-Soriguer Solanas, F., Pérez Latorre, A. V. \& Cabezudo, B. (2019). Novedades para la flora vascular del Espacio Natural Sierra de las Nieves (Málaga, España), Acta Botanica Malacitana, 44, 117-124.

http://dx.doi.org/10.24310/abm.v44i0.6400

Castroviejo, S. (Coord.) (1986-2019). Flora iberica 1-18, 20, 21. Madrid, España: CSIC.

Castroviejo, S. \& Velayos, M. (1997). Sedum L. In S. Castroviejo, C. Aedo, M. Laínz, R. Morales, F. Muñoz Garmendia, G. Nieto Feliner \& J. Paiva (Eds.), Flora iberica 5 (pp. 123-152). Madrid, España: CSIC.

DERA [2019]. Datos Espaciales de Referencia de Andalucía. Recurso electrónico en www.juntadeandalucia.es/institutodeestadisticayc artografia./DERA/index.htm. Consulta realizada en febrero de 2019.

Ferrer Gallego, P.P. \& Guara Requena, M. (2007). Especies del género Gagea Salisb. (Liliaceae) presentes en el levante peninsular ibérico. Lagascalia, 27, 31-51.

GBIF [2019]. Global Biodiversity Information Facility. Recurso electrónico en www.gbif.es. Consulta realizada en agosto de 2019.

Hernández Bermejo, J. E. \& Clemente, M., (1993). Lepidium L. In S. Castroviejo, C. Aedo, C. Gómez Campo, M. Laínz, P. Montserrat, R. Morales, F.
Muñoz Garmendia, G. Nieto Feliner, E. Rico, S. Talavera \& L. Villar (Eds.), Flora iberica 4 (pp. 311-327). Madrid, España: CSIC.

Lidén, M., (1986). Sarcocapnos L. In S. Castroviejo, M. Laínz, G. López González, P. Montserrat, F. Muñoz Garmendia, J. Paiva \& L. Villar (Eds.), Flora iberica 1 (pp. 433-438). Madrid, España: CSIC.

López González, G. (1986). Ophioglossum L. In S. Castroviejo, M. Laínz, G. López González, P. Montserrat, F. Muñoz Garmendia, J. Paiva \& L. Villar (Eds.), Flora iberica 1 (pp. 36-37). Madrid, España: CSIC.

López González, G. (2013). Gagea L. In E. Rico, M.B. Crespo, A. Quintanar, A. Herrero, \& C. Aedo (Eds.), Flora iberica 20 (pp. 22-74). Madrid, España: CSIC.

Lorite, J. (2016). An updated checklist of the vascular flora of Sierra Nevada (SE Spain). Phytotaxa, 261(1), 1-57. doi: http://dx.doi.org/10.11646/phytotaxa.261.1.1

Lorite, J., Ruiz Girela, M. \& Donaire, F. (2005). Datos sobre flora endémica, rara o amenazada de Sierra Nevada (SE. España). Acta Botanica Malacitana, 30, 237-239

Martínez Parras, J.M., Peinado Lorca, M. \& Alcaraz Ariza, F. (1985). Datos sobre la vegetación de Sierra Nevada. Lazaroa, 7, 515-533.

Marfil, J. M., Molero, J., Cantó, P. \& Rivas-Martínez, S., (2016). Bioindicators and bioclimatic data as essential tools towards a consistent biogeographic district typology of Sierra Nevada National Park (Spain). Lazaroa, 38(1), 7-25. doi: http://dx.doi.org/10.5209/LAZAROA.47929

Molero Mesa, J. \& Martínez Parras, J.M. (1983). Plantas de Sierra Nevada. Anales del Jardin Botanico de Madrid, 40(1), 171-175.

Molero Mesa, J. \& Pérez Raya, F. (1987). La flora de Sierra Nevada. Granada, España: Diputación de Granada.

Monserrat Martí, J. M., (1990). Moehringia L. In S. Castroviejo, M. Laínz, G. López González, P. Montserrat, F. Muñoz Garmendia, J. Paiva \& L. Villar (Eds.), Flora iberica 2 (pp. 225-230). Madrid, España: CSIC.

Morales Abad, M.J. (1990). Asientos para un Atlas Corológico de la Flora Occidental. Mapa 390. Fontqueria, 28, 82-84.

Morales, M.J. \& Fernández Casas, J. (1989): Asientos para un Atlas Corológico de la Flora Occidental. Mapa 284. Fontqueria, 25, 38-40.

Morales, C. \& Romero-García, A.T. (1991). A new species of the genus Sarcocapnos (Fumariaceae) from eastern Andalusia (Spain). Plant Systematics and Evolution, 177, 1-10.

Navarro, F.B., Jiménez, M.N. \& Salazar, C. (2007). Contribución al catálogo florístico del Parque Natural de la Sierra de Baza (Granada, España), II. Acta Botanica Malacitana, 32, 285-289.

Negrillo, A. M. (2011). Elymus L. In G. Blanca, B. Cabezudo, M. Cueto, C. Morales Torres \& C. Salazar (Eds.), Claves de la Flora Vascular de Andalucía Oriental, (pp. 121-122). Granada, 
España: Universidades de Almería, Granada, Jaén y Málaga.

Nieto Feliner, G. (1997). Epilobium L. In S. Castroviejo, C. Aedo, C. Benedí, M. Laínz, F. Muñoz Garmendia, G. Nieto Feliner \& J. Paiva (Eds.), Flora iberica 8, (pp.101-131). Madrid, España: CSIC.

Pavón Núñez, M. \& Pérez Latorre, A.V. (2010). Sobre las avellanedas subbéticas con Ulmus glabra Huds. en las sierras de Cazorla-SeguraAlcaraz, Jaén-Albacete (España). Lagascalia, 30, 393-405.

Pavón Núñez, M., Pérez Latorre, A.V. \& Hidalgo Triana N. (2015). Novedades fitosociológicas y florísticas en el Parque Natural de Tejeda-Almijara y Alhama (Málaga-Granada, España). Acta Botanica Malacitana, 40, 199-205.

Peñas, J., Pérez-García, F.J. \& Mota, J.F. (2005). Patterns of endemic plants and biogeography of the Baetic high mountains (south Spain). Acta Botanica Gallica, 152 (3), 347-360.

Pérez Latorre, A.V., Navas Fernández, D., Gavira, O., Caballero, G. \& Cabezudo, B. (2004). Vegetación del P. N. de las Sierra Tejeda, Almijara y Alhama. Acta Botanica Malacitana, 29, 117-190.

Pérez Latorre, A.V., Pavón Núñez, M. \& Hidalgo Triana, N. (2011). Sobre las alisedas nevadenses (Sierra Nevada, Granada-Almería, España). Lagascalia, 31, 161-174.

Pérez García, F.J., Cueto, M., Peñas, J., Martínez Hernández, F., Medina Cazorla, J.M., Garrido Becerra, J.A. \& Mota, J.F. (2007). Selection of an endemic flora reserve network and its biogeographical significance in the Baetic ranges (Southern Spain). Acta Botanica Gallica, 154(4), 545-571.

Prieto, P. (1975). Los bosques de Sierra Nevada, Anales del Instituto Botánico Cavanilles, 32 (2), 1099-1129.

Rigueiro Rodríguez, A. (1978). Catalogo florístico de la Sierra de Grazalema. Trabajo de la Catedra de Botanica de la Escuela Tecnica Superior de Ingenieros de Montes, 4, 1-272.Ríos Ruiz, S., Alcaraz Ariza, F. \& Valdés Franzi, A. (2003). Vegetación de sotos y riberas de la provincia de Albacete (España). Albacete, España: Diputación de Albacete.

Rivas Martínez, S. (1961). Los pisos de la vegetación de la Sierra Nevada. Boletín de la Real Sociedad Española de Historia Natural, Secc. Biol., 59, 55-64.

Rivas Martínez, S. (2011). Mapa de series, geoseries y geopermaseries de vegetación de España. Memoria del Mapa de Vegetación Potencial de España. Parte 1. Itinera Geobotanica, 18(1), 5-424.

Salazar, C. (1996). Estudio Fitosociológico de la Vegetación Riparia Andaluza (Provincia Bética): Cuenca del Guadiana Menor (Tesis doctoral). Universidad de Jaén, Jaén, España.

Sánchez Gómez, P., López Espinosa, J.A., Vera, J.B., López Romero, C. \& Jiménez, J. F. (2005). Novedades corológicas para la flora vascular del sureste ibérico. Anales de Biología, 27, 127-132.

SIVIM [2019]. Sistema de información de la vegetación lbérica y Macaronésica. Recurso electrónico disponible en www.sivim.info/sivi/. Consulta realizada en agosto de 2019.

Socorro, O. (1982). Dos nuevas citas para la provincia de Granada. Anales del Jardín Botánico de Madrid, 38(2), 538.

Soriano, C. (1983). Contribución al catálogo florístico del macizo de Segura-Cazorla (Andalucía, España). Fontqueria, 3, 1-5.

Valle, F., Gómez-Mercado, F. \& Mota, J.F. (1988). Los robledales de la Sierra de Segura y otras comunidades relacionadas con ellos. Anales del Jardín Botánico de Madrid, 45(1), 247-257.

Varo, J. \& Salvo, A.E. (1982). Ensayo biogeográfico sobre la pteridoflora de Sierra Nevada (Granada, España). Acta Botanica Malacitana, 7, 203-210. 